\title{
Vibrotactile Sensitivity of the Glabrous Hand and Perioral Face in Neurotypical Children and Adults
}

\author{
Elizabeth C Sandfort ${ }^{1}$, Jaehoon Lee ${ }^{2}$, Jacob Greenwood ${ }^{3}$ and Steven M Barlow*4 \\ ${ }^{1}$ Department of Special Education and Communication Disorders, Communication Neuroscience Laboratories, University of \\ Nebraska, USA
}

${ }^{2}$ Department of Educational Psychology \& Leadership, Texas Tech University, USA

${ }^{3}$ Communication Neuroscience Laboratories, Department of Biological Systems Engineering, University of Nebraska, USA

${ }^{4}$ Department of Special Education and Communication Disorders, Department of Biological Systems Engineering, Communication Neuroscience Laboratories, University of Nebraska, USA

*Corresponding author: Steven M Barlow, Department of Special Education and Communication Disorders, Department of Biological Systems Engineering, Communication Neuroscience Laboratories, University of Nebraska,141 Barkley Memorial Center, Lincoln, NE 68583, USA

\begin{abstract}
ARTICLE INFO
Received: 蔧 April 28, 2021

Published: 幽 May 18, 2021

Citation: Elizabeth C Sandfort, Jaehoon Lee, Jacob Greenwood, Steven M Barlow. Vibrotactile Sensitivity of the Glabrous Hand and Perioral Face in Neurotypical Children and Adults. Biomed J Sci \& Tech Res 35(5)-2021. BJSTR. MS.ID.005768.
\end{abstract}

Keywords: Somatosensory; Adaptive Tactile Threshold Tracking; Glabrous Finger; Nonglabrous Perioral Face; Children; Adults

Abbreviations: VDT: Vibrotactile Detection Threshold; DVRT: Differential Variable Reluctance Transformer; LMM: Linear Mixed Modeling

\section{ABSTRACT}

Purpose: Experimental findings are limited concerning tactile sensitivity of the glabrous hand and perioral face in neurotypical children. Additional research examining vibrotactile detection thresholds (VDT) in neurotypical children would further understanding of tactile perception in children and add to our understanding of somatosensory development across the lifespan.

Method: A microprocessor-controlled, automated single-interval up/down (SIUD) adaptive tracking system, including stimulus control and perceptual response logging, was used to estimate vibrotactile detection thresholds (VDT) bilaterally for the glabrous index finger and perioral hairy skin at the oral angle in two neurotypical cohorts, including 37 children (age 10-13 years) and 45 adults (age 19-35 years) in response to sinusoidal mechanical stimuli presented at 5, 10, 50, 150, 250, and $300 \mathrm{~Hz}$.

Results: Linear mixed modeling (LMM) analysis revealed that the effects of skin site $(\mathrm{F}=115.74, \mathrm{p}<0.0001)$ and stimulus frequency $(\mathrm{F}=146.42, \mathrm{p}<0.0001)$ were significant, whereas the effects of $\operatorname{sex}(F=0.83, p=0.363)$ and age group $(F=2.11, p=0.1507)$ were not significant. The increased sensitivity (classic U-function) at $250 \mathrm{~Hz}$, attributable to the presence of the rapidly adapting Pacinian corpuscles in the glabrous hand, was not apparent in the perioral vibrogram. The absolute threshold values for perioral skin were higher and the magnitude of VDT differences was greater across stimulation frequency compared to glabrous finger VDTs. No significant differences in VDT were apparent between left and right skin sites regardless of sex and age group.

Conclusion: The somatosensory hand and perioral face show similar tactile acuity in response to sinusoidal mechanical stimuli ranging from 5 to $300 \mathrm{~Hz}$ in preadolescent children and adults. The automated SIUD adaptive VDT tracking algorithm provides clinicians with a reliable tool for rapid assessment of the cutaneous somatosensory system across the lifespan. 


\section{Introduction}

Neural encoding of tactile features by the somatosensory system serves many important perceptual roles, including texture and object discrimination, feedback for precision grip and manipulation, and orofacial motor control for speech, gesture, and eating. Tactile feature encoding also plays a central role in haptics and motor development, and maintenance of perceptual skills and fine motor control as the somatic body plan grows from infancy into adulthood. These rapidly conducting somatosensory channels also play a key role in activity- and experience-dependent cortical neuroplasticity and recovery of motor function following injury to the brain (e.g., cerebrovascular stroke). While vibrotactile threshold testing has been used for nearly 100 years [1], data are limited concerning tactile sensitivity of the glabrous hand and perioral face in neurotypical children. Therefore, the aim of this study was to expand the knowledge base of vibrotactile detection thresholds (VDT) in neurotypical children [age 10-13 years] and compare to adults [age 19 to 35 years] assessed using an automated adaptive single interval up-down (SIUD) algorithm in response to randomized blocks of sinusoidal stimulation $(5,10,50,150,250$, and $300 \mathrm{~Hz}$ ) applied to the skin of the glabrous hand and perioral face.

\section{A Comparison of VDTs of the Hand and Orofacial Structures}

Recent studies have compared VDTs between orofacial structures and the hand over a range of vibratory frequencies $[2,3]$. In a seminal study on orofacial somatic sensation [4], an ascending method of limits was used to estimate VDTs sampled from 12 neurotypical adult participants (mean age 19.25 years) for five areas on the face including the chin, lower lip (hairy skin), lip vermilion, oral angle, and the cheek, and a single site on the glabrous hand (right D2 digit). A linear motor equipped with a nylon center probe $(0.5 \mathrm{~cm} 2$ area) and annular surround ( $1 \mathrm{~mm}$ gap) was used to deliver ramped mechanical sinusoidal stimuli (1 second $\mathrm{ON}, 1$ second OFF) at discrete test frequencies [5, 10, 50, 150, 250, 300, 400, $600 \mathrm{~Hz}$ ]. Mean VDTs for all facial skin sites were significantly higher than thresholds for the index finger. Pacinian-type sensitivity with the classic U-function, characteristic of the glabrous finger [5], was absent in the face. Pacinian corpuscles are notably absent in the orofacial structures of non-human primates, except for the ventral tongue surface [6]. The PC dip for the glabrous finger occurred at $250 \mathrm{~Hz}$ and is consistent with previous psychophysical work [5].

Compared to the glabrous finger, VDTs in the lower face were significantly elevated and flattened from 150 to $600 \mathrm{~Hz}$ [4]. A subsequent study of VDT sensitivity of the upper and lower lip using an adaptive threshold tracking algorithm found significant main effects for test frequency and stimulation site [7]. Lip vermilion skin sites near midline yielded lower VDTs compared to lateral sites on the lips. The best frequency yielding the lowest VDTs for all test sites was $50 \mathrm{~Hz}$. The effects of age and sex on VDTs have been explored in two cohorts of adults using the same instrumentation [3]. Vibrotactile thresholds were measured at six test frequencies $[5,10,50,150,250,300 \mathrm{~Hz}]$ in the face and hand of eighteen young adults (age 23.8 years) and eighteen older adults (age 62.2 years). VDTs were measured for the right lower lip vermilion, right nonglabrous oral angle, and glabrous right D2 using a single interval up/down (SIUD) adaptive threshold tracking procedure [8].

VDTs were significantly dependent on stimulus frequency, skin site, and sex. As expected, the glabrous index finger yielded the lowest threshold and manifest the classic U-function with acute sensitivity at $250 \mathrm{~Hz}$. Diminished sensitivity to vibrotactile stimulation was apparent in the older cohort for the glabrous finger (D2) between 5 and $300 \mathrm{~Hz}$. Since the hands are used often to manipulate objects and perform work, they are at increased risk use or work-related damage to the glabrous skin, including primary mechanoreceptive afferents and the surrounding integument [3]. The current study will provide new information about VDTs in male and female children across a broad range of discrete test frequencies spanning 5 to $300 \mathrm{~Hz}$, for comparison to similar measures obtained in adults using the automated SIUD adaptive VDT algorithm $[2,8,9]$. The SIUD tracking method as described in the present report generates estimates of tactile thresholds at 6 test frequencies in $\sim 3$ minutes 45 seconds per skin site.

An efficient psychophysical tracking algorithm is advantageous for VDT estimation in challenging pediatric and other clinical populations where vigilance may be an issue. These data will add to our understanding of somatosensory vibrotactile sensitivity from childhood through age 35 years. Moreover, expanded application of VDT measures has clear clinical applications in children and adults with neurological issues, including peripheral mechanosensory neuropathy, and central somatosensory processing disorders [9$14]$.

\section{Hypotheses}

Independent variables in this study included vibratory stimulation frequency, age group, sex, and stimulus site. The dependent variable was vibrotactile detection threshold. Significant main effects were predicted for stimulus frequency with adults manifesting significantly lower thresholds compared to children [15]. As for sex, it was hypothesized that there would be significant sex differences for VDTs among children and adults, inspired by our previous findings in young adult subjects [3]. For stimulus site, it was hypothesized that the glabrous index finger would yield a clear advantage with lower VDTs compared to the commissural (hairy) skin at the oral angle of the lower face, consistent with observations in young adults [2-4]. 


\section{Materials and Methods}

\section{Participants}

Study participants included 37 neurotypical preadolescent children ages 10-13 years (Mean age $11.41 \mathrm{yrs}, 15 \mathrm{~F} / 22 \mathrm{M}$ ) and 45 neurotypical adults ages 19-35 years (Mean age 23.98 yrs, 22F/23M). Participants were recruited regardless of ethnicity or race. Written informed consent was obtained from adult participants, whereas both child assent and parent/legal guardian written consent were obtained for minors. The Institutional Review Board of the University of Nebraska-Lincoln approved all consent/ assent documentation (IRB \#18912). Inclusion criteria: no report of neurological injury or illness. Exclusion criteria: neurologic disease-causing sensorimotor impairment to the face and/or hand, traumatic injury to the hand or face causing sensorimotor impairment.

\section{Instrumentation and Data Collection}

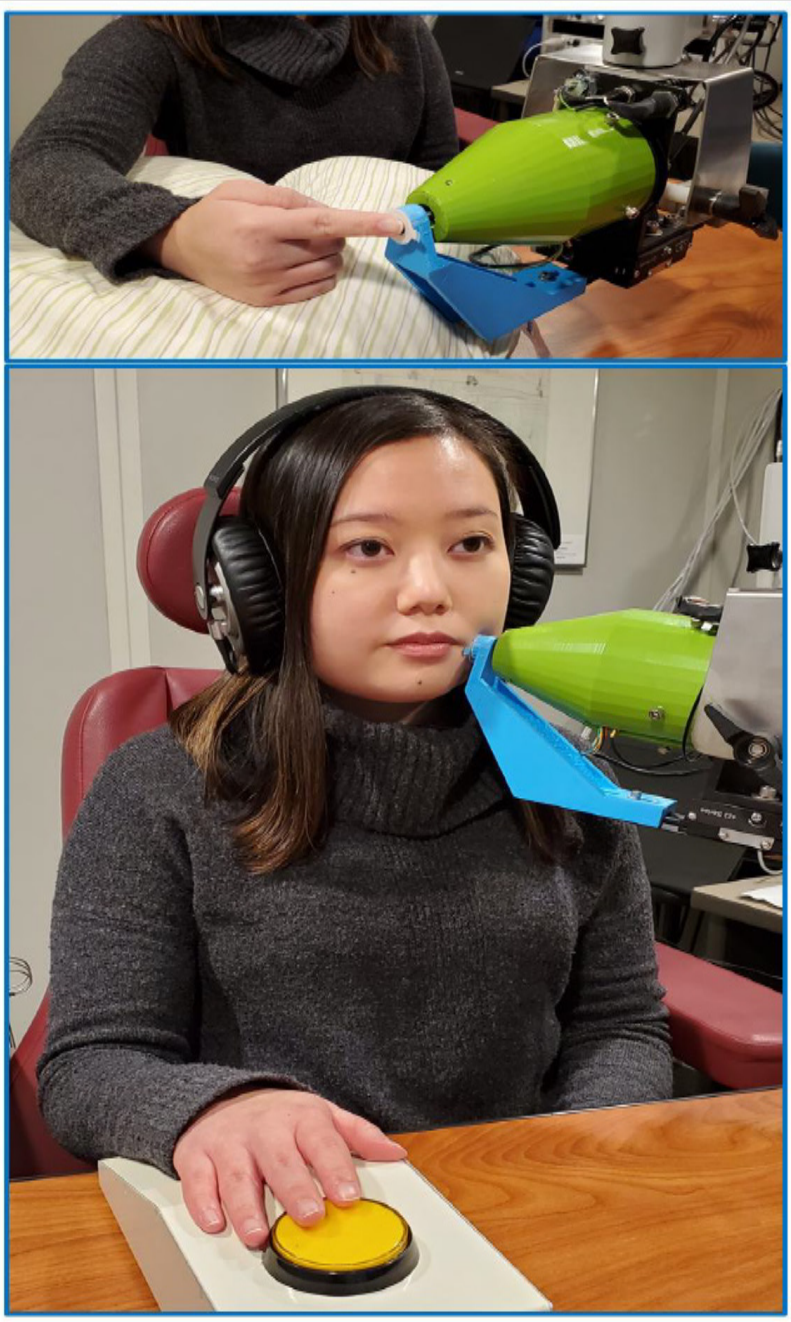

Figure 1: Test configuration for sampling vibrotactile detection thresholds at the glabrous index fingertip and oral angle.
VDTs for each participant were evaluated using an automated mechanical stimulus control and threshold detection system developed in our laboratory [3]. A linear motor (Brüel \& Kjaer Minishaker, model 4810) was used to deliver mechanical oscillatory stimuli to the skin at frequencies from 5 to $300 \mathrm{~Hz}$. The sinusoidal bursts (100 millisecond linear rise-fall decay, 1 second duration) were synthesized by a National Instruments cRIO realtime field-programmable gate array (FPGA) embedded controller programmed in LabVIEW. The generated burst was conditioned with a DC-coupled power amplifier (Brüel \& Kjaer model 2706) and input to the Minishaker (Figure 1). Specialized fixtures and sensors coupled to the Minishaker included a nylon contractor probe $\left(8 \mathrm{~mm}\right.$ diameter, surface area $\left.=0.5 \mathrm{~cm}^{2}\right)$, a Schaevitz microminiature displacement sensor (DC-800Hz), and a $19 \mathrm{~mm}$ diameter rigid annular surround coupled to a linear translation stage. The surround was adjusted for a $1000 \mu \mathrm{m}$ preload.

The stimulator probe was placed against the surface of the skin of the lower face and glabrous hand. The surround was secured to the skin with a double adhesive collar. The participant was seated in a dental examination chair and instructed to press a response button when they felt the vibratory stimulus on their lower face or hand. Narrow-band noise [66-68 dB(C) SPL] was presented to each participant through circumaural headphones to mask the potential acoustic emittance associated with the Minishaker at test frequencies $\geq 50 \mathrm{~Hz}$. Vibratory stimuli were presented in randomized blocks at $5,10,50,150,250$, and $300 \mathrm{~Hz}$ to either the lower face or glabrous distal surface of the index finger, D2. Skin site was randomized among participants.

\section{Adaptive Psychophysical Threshold Tracking Procedure}

A single interval up/down (SIUD) adaptive procedure based on eight trials to estimate VDT [8] was used for VDT estimation. The SIUD procedure begins with a suprathreshold stimulus to orient the participant and elicit a positive button press response. Initial step size was $10 \mathrm{~dB}$, and subsequent step sizes were $\pm 5 \mathrm{~dB}$ relative to the amplitude of the first trial. Following a negative response, step size was reduced to $2 \mathrm{~dB}$ for the remaining trials. The starting point for the 8 trials was the trial before the first negative response. The algorithm incorporated false positive trials as foils. For example, if a false positive was detected (e.g., participant responded when in fact no stimulus was presented), the data were discarded, and a new trial was initiated [3].

\section{Statistical Analysis}

Linear mixed modeling (LMM) was conducted to compare vibrotactile threshold between frequency $(5,10,50,150,250$, $300 \mathrm{~Hz}$ ), skin surface (left- and right-index finger, left and right oral angle), sex (male, female), and age group (children, adults), and examine potential interactions of those conditions. The models accounted for nesting of repeated measurements within participants 
(i.e., intraclass correlation), thereby providing unbiased estimates for condition differences. When an overall difference was significant across conditions, marginal means were pairwise compared at a Bonferroni-corrected alpha level while controlling for Type I error at the nominal level. A proper error covariance structure was determined based on model fit (i.e., adjusted Akaike Information Criterion, Bayesian Information Criterion). All statistical analyses were conducted using SAS 9.4 [16].

\section{Results}

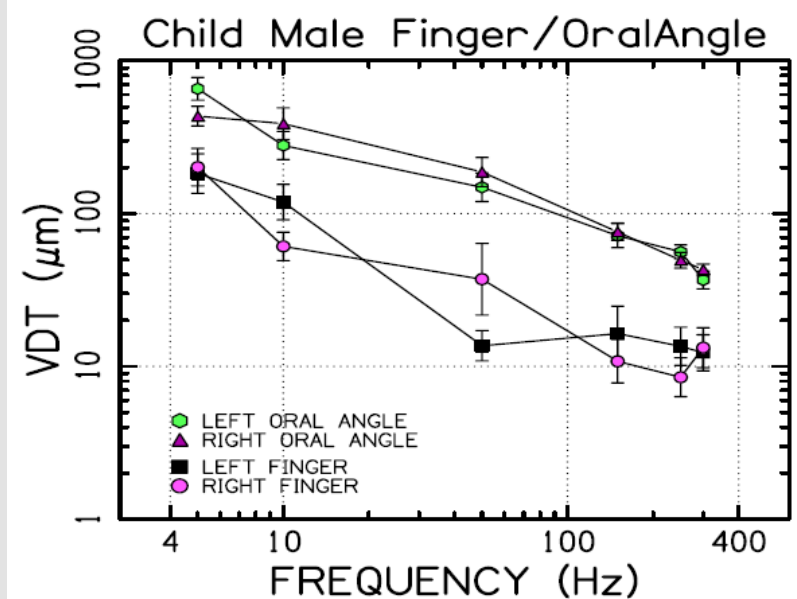

Figure 2: Vibrotactile detection thresholds for 22 male children.

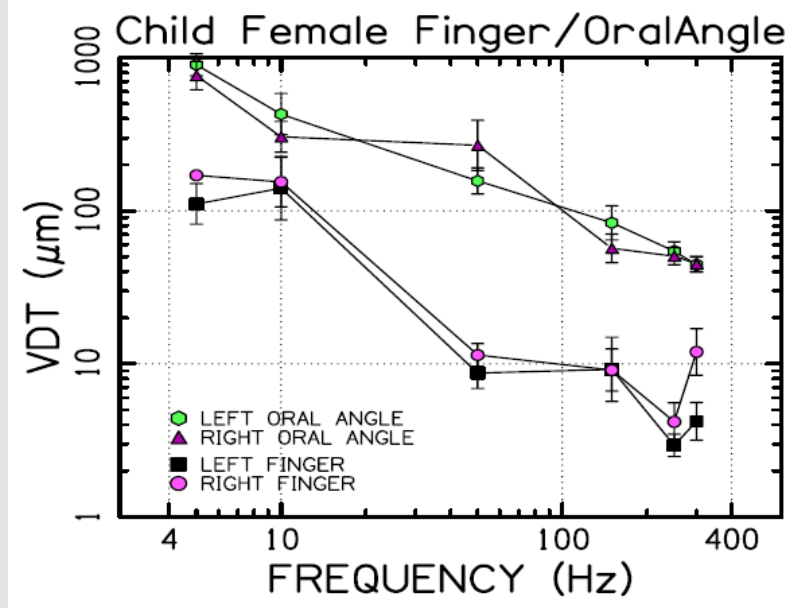

Figure 3: Vibrotactile detection thresholds for 15 female children.

VDTs were sampled from 37 children and 45 adults. Bilateral, glabrous index finger and oral angle VDTs are plotted in log-log scales in (Figures 2) through 5 according to sex (male, female) and age group (child, adult). Overall, VDT functions for the oral angle commissure and glabrous index finger showed decreasing thresholds as function of stimulus frequency. The glabrous index finger had the clear advantage in vibrotactile threshold sensitivity compared to the lower face at all test frequencies. The PC dip, attributed to high frequency sensitivity of the Pacinian corpuscle, was evident for the index finger at $250 \mathrm{~Hz}$, whereas the VDT was at least an order of magnitude greater for vibratory stimuli delivered to the oral angle commissure which lacks PC mechanoreceptors. The marginal means (adjusted for sex and age group in LMM) for skin site, stimulus frequency, and skin site by stimulus frequency are shown in (Figures 3-8), respectively. The descriptive statistics for VDTs by sex, age group, skin site, and test frequency are given in (Tables 1-4).

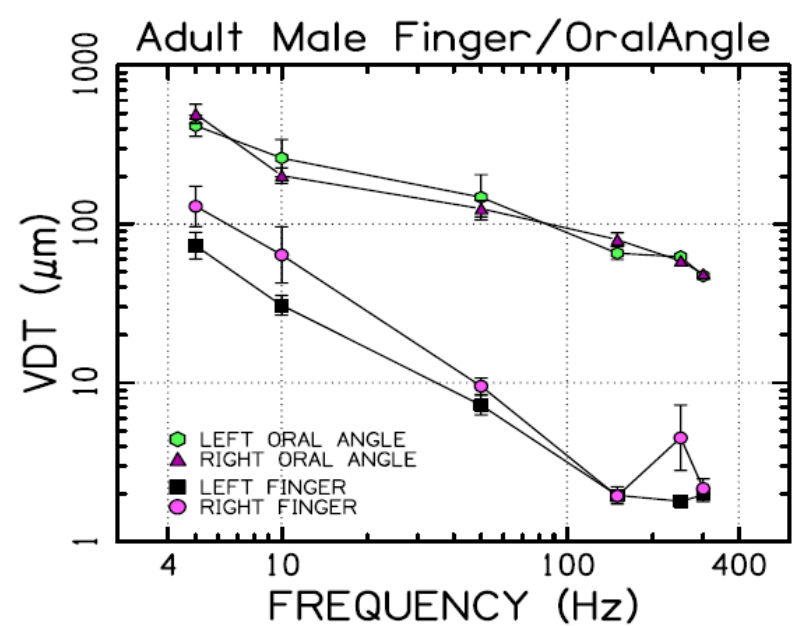

Figure 4: Vibrotactile detection thresholds for 23 adult males.

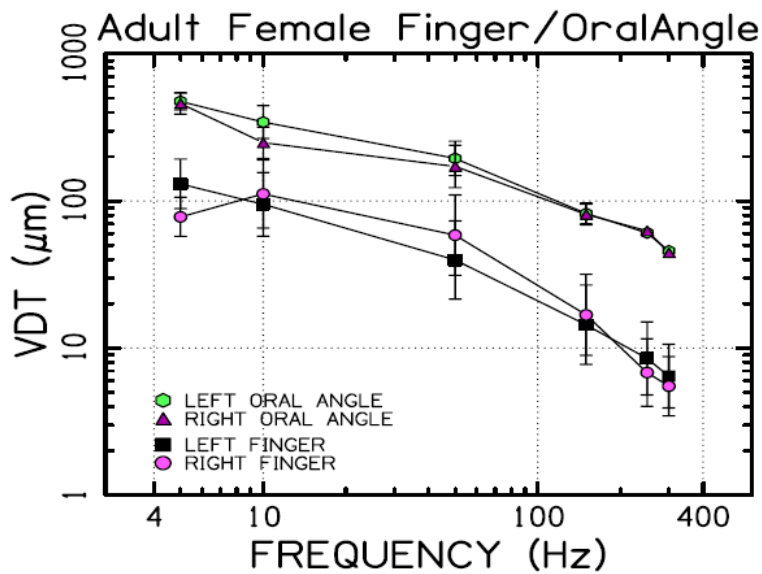

Figure 5: Vibrotactile detection thresholds for 22 adult females. 


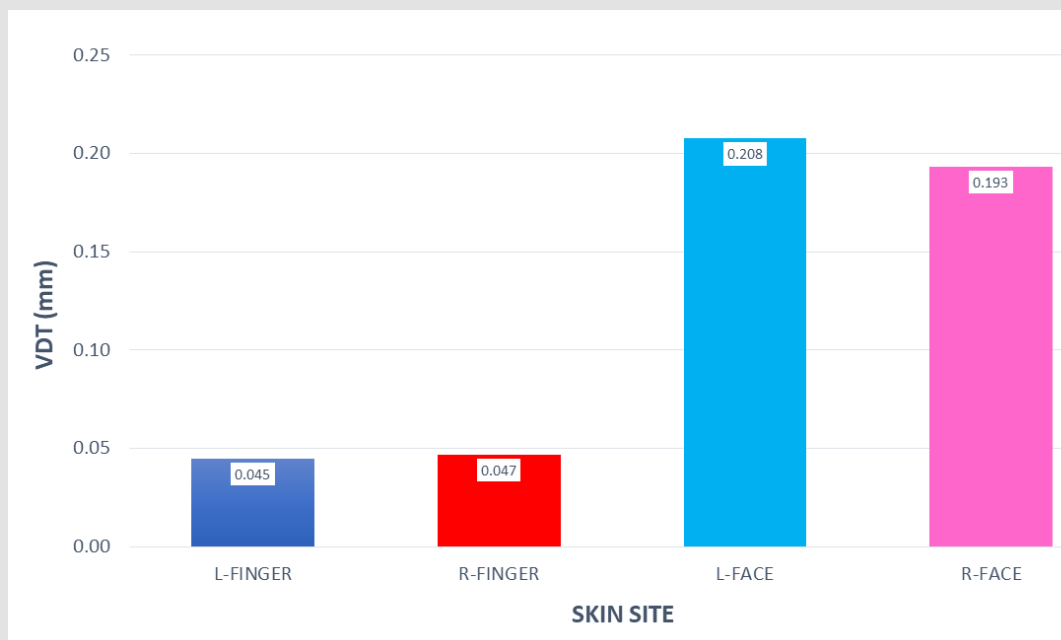

Figure 6: Marginal means for VDT as a function of 'skin site.'

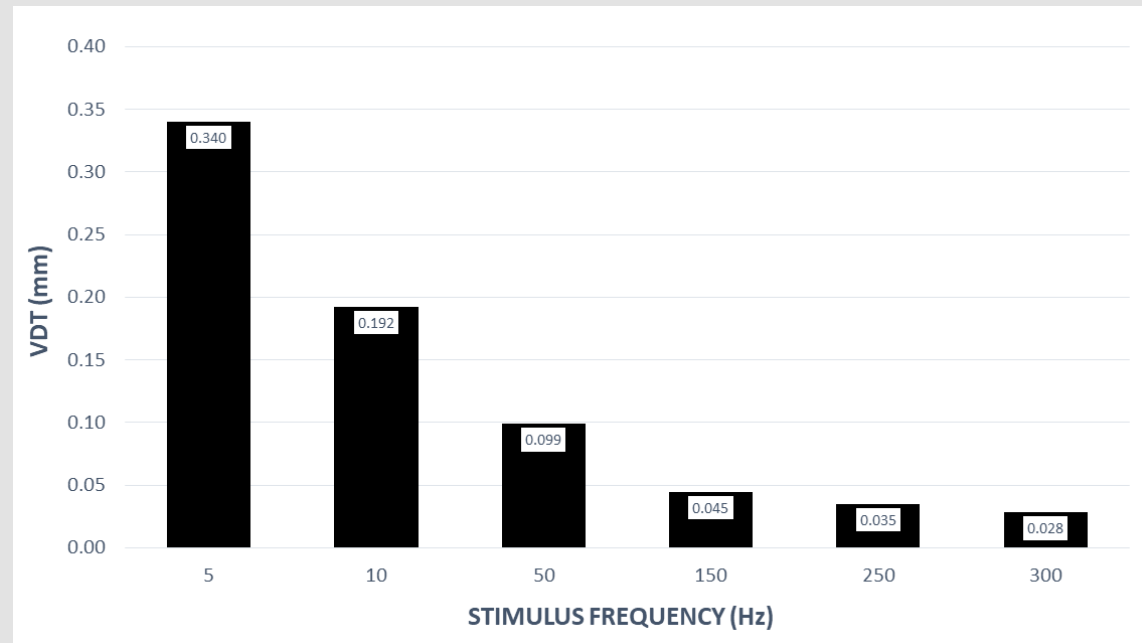

Figure 7: Marginal means for VDT as a function of 'stimulus frequency $(\mathrm{Hz})$ '.

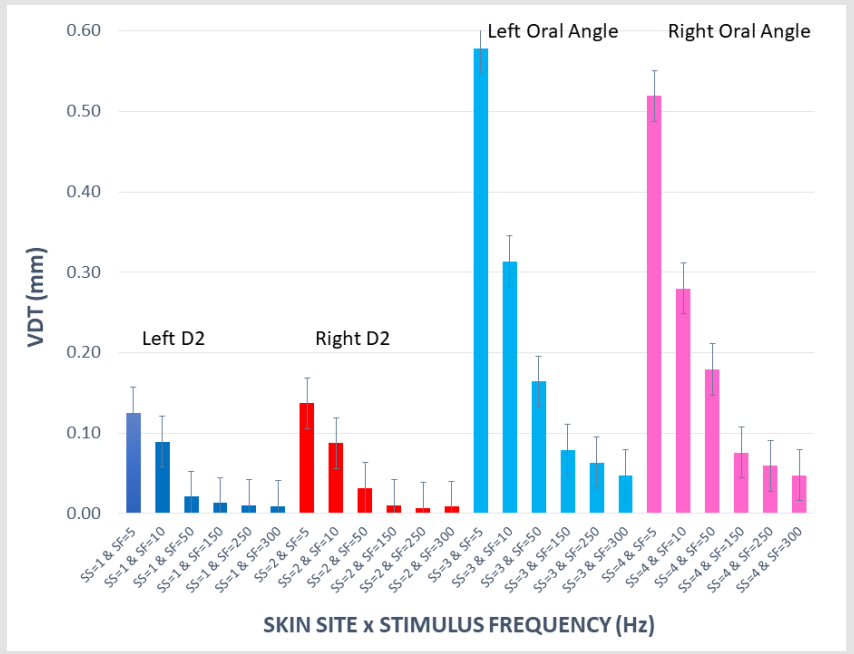

Figure 8: Marginal means for VDT expressed in millimeters as a function of 'skin site' by 'stimulus frequency (Hz)'. 
Table 1: Child female ( $\mathrm{N}=15)$ VDT descriptive statistics. Acceleration: $\mathrm{a}=-2 \mathrm{n} 2 \mathrm{f} 2 \mathrm{D} \sin (2 \mathrm{nft})$, where $\mathrm{D}$ is displacement (mm), and $\mathrm{f}$ is frequency $(\mathrm{Hz})$.

\begin{tabular}{|c|c|c|c|c|}
\hline \multicolumn{5}{|c|}{ CHILD FEMALE } \\
\hline \multicolumn{5}{|c|}{ Left Finger } \\
\hline FREQ(Hz) & VDT $(\mathrm{mm})$ & S.E. MEAN & S.D. MEAN & Accel(peak) [g] \\
\hline 5 & 0.111000 & 0.03960 & 0.125300 & 0.00559 \\
\hline 10 & 0.140400 & 0.08540 & 0.270000 & 0.02826 \\
\hline 50 & 0.008700 & 0.00229 & 0.007250 & 0.04378 \\
\hline 150 & 0.009200 & 0.00573 & 0.018130 & 0.41666 \\
\hline 250 & 0.002931 & 0.00054 & 0.001708 & 0.36873 \\
\hline 300 & 0.004210 & 0.00138 & 0.004370 & 0.76266 \\
\hline \multicolumn{5}{|c|}{ Right Finger } \\
\hline FREQ(Hz) & VDT(mm) & S.E. MEAN & S.D. MEAN & Accel(peak) [g] \\
\hline 5 & 0.17080 & 0.07970 & 0.26440 & 0.00859 \\
\hline 10 & 0.15430 & 0.06970 & 0.23110 & 0.03106 \\
\hline 50 & 0.01142 & 0.00214 & 0.00709 & 0.05747 \\
\hline 150 & 0.00911 & 0.00340 & 0.01129 & 0.41258 \\
\hline 250 & 0.00418 & 0.00138 & 0.00458 & 0.52585 \\
\hline 300 & 0.01197 & 0.00502 & 0.01665 & 2.16843 \\
\hline \multicolumn{5}{|c|}{ Left Oral Angle } \\
\hline FREQ(Hz) & VDT(mm) & S.E. MEAN & S.D. MEAN & Accel(peak) [g] \\
\hline 5 & 0.90100 & 0.16100 & 0.50800 & 0.04534 \\
\hline 10 & 0.42800 & 0.15600 & 0.49300 & 0.08615 \\
\hline 50 & 0.15680 & 0.03420 & 0.10800 & 0.78903 \\
\hline 150 & 0.08350 & 0.02460 & 0.07790 & 3.78162 \\
\hline 250 & 0.05449 & 0.00824 & 0.02607 & 6.85498 \\
\hline 300 & 0.04449 & 0.00532 & 0.01682 & 8.05961 \\
\hline \multicolumn{5}{|c|}{ Right Oral Angle } \\
\hline $\operatorname{FREQ}(\mathrm{Hz})$ & VDT (mm) & S.E. MEAN & S.D. MEAN & Accel(peak) [g] \\
\hline 5 & 0.76500 & 0.18200 & 0.63200 & 0.03850 \\
\hline 10 & 0.30480 & 0.07940 & 0.27510 & 0.06135 \\
\hline 50 & 0.26800 & 0.12300 & 0.42600 & 1.34860 \\
\hline 150 & 0.05690 & 0.01380 & 0.04780 & 2.57694 \\
\hline 250 & 0.05056 & 0.00689 & 0.02386 & 6.36057 \\
\hline 300 & 0.04503 & 0.00558 & 0.01934 & 8.15743 \\
\hline
\end{tabular}

Table 2: Child male $(\mathrm{N}=22)$ VDT descriptive statistics. Acceleration: $a=-2 \Pi 2 f 2 D \sin (2 \pi f t)$, where $D$ is displacement (mm), and $f$ is frequency $(\mathrm{Hz})$.

\begin{tabular}{|c|c|c|c|c|}
\hline \multicolumn{5}{|c|}{ CHILD MALE } \\
\hline \multicolumn{7}{|c|}{ Left Finger } \\
\hline FREQ(Hz) & VDT(mm) & S.E. MEAN & S.D. MEAN & Accel(peak) [g] \\
\hline 5 & 0.18270 & 0.06290 & 0.29500 & 0.00942 \\
\hline 10 & 0.11890 & 0.03620 & 0.17000 & 0.02393 \\
\hline 50 & 0.01365 & 0.00350 & 0.01641 & 0.06869 \\
\hline 150 & 0.01640 & 0.00839 & 0.03937 & 1.70337 \\
\hline 300 & 0.01354 & 0.00448 & 0.02100 & 2.22640 \\
\hline
\end{tabular}




\begin{tabular}{|c|c|c|c|c|}
\hline \multicolumn{5}{|c|}{ Right Finger } \\
\hline FREQ(Hz) & $\operatorname{VDT}(\mathrm{mm})$ & S.E. MEAN & S.D. MEAN & Accel(peak) [g] \\
\hline 5 & 0.20150 & 0.06600 & 0.30980 & 0.01014 \\
\hline 10 & 0.06100 & 0.01450 & 0.06810 & 0.01228 \\
\hline 50 & 0.03720 & 0.02660 & 0.12490 & 0.18719 \\
\hline 150 & 0.01081 & 0.00415 & 0.01947 & 0.48957 \\
\hline 250 & 0.00849 & 0.00288 & 0.01349 & 1.06806 \\
\hline 300 & 0.01326 & 0.00465 & 0.02182 & 2.40212 \\
\hline \multicolumn{5}{|c|}{ Left Oral Angle } \\
\hline FREQ(Hz) & VDT(mm) & S.E. MEAN & S.D. MEAN & Accel(peak) [g] \\
\hline 5 & 0.65600 & 0.12200 & 0.57200 & 0.03301 \\
\hline 10 & 0.27930 & 0.06560 & 0.30750 & 0.05622 \\
\hline 50 & 0.14870 & 0.03570 & 0.16750 & 0.74827 \\
\hline 150 & 0.07170 & 0.01420 & 0.06680 & 3.24721 \\
\hline 250 & 0.05613 & 0.00618 & 0.02896 & 7.06129 \\
\hline 300 & 0.03685 & 0.00524 & 0.02457 & 6.67558 \\
\hline \multicolumn{5}{|c|}{ Right Oral Angle } \\
\hline FREQ(Hz) & $\operatorname{VDT}(\mathrm{mm})$ & S.E. MEAN & S.D. MEAN & Accel(peak) [g] \\
\hline 5 & 0.43440 & 0.06940 & 0.32540 & 0.02186 \\
\hline 10 & 0.38800 & 0.10400 & 0.48800 & 0.07810 \\
\hline 50 & 0.18700 & 0.04560 & 0.21400 & 1.07687 \\
\hline 150 & 0.07600 & 0.01050 & 0.04920 & 3.44195 \\
\hline 250 & 0.04951 & 0.00630 & 0.02957 & 6.22848 \\
\hline 300 & 0.04299 & 0.00372 & 0.01744 & 7.78788 \\
\hline
\end{tabular}

Table 3: Adult female ( $\mathrm{N}=22)$ VDT descriptive statistics. Acceleration: $\mathrm{a}=-2 \mathrm{n} 2 \mathrm{f} 2 \mathrm{D} \sin (2 \mathrm{nft})$, where $\mathrm{D}$ is displacement (mm), and $\mathrm{f}$ is frequency $(\mathrm{Hz})$.

\begin{tabular}{|c|c|c|c|c|}
\hline \multicolumn{5}{|c|}{ ADULT FEMALE } \\
\hline \multicolumn{5}{|c|}{ Left Finger } \\
\hline FREQ(Hz) & $\operatorname{VDT}(\mathrm{mm})$ & S.E. MEAN & S.D. MEAN & Accel(peak) [g] \\
\hline 5 & 0.13060 & 0.06170 & 0.28950 & 0.00657 \\
\hline 10 & 0.09490 & 0.06150 & 0.28840 & 0.01910 \\
\hline 50 & 0.03970 & 0.03350 & 0.15710 & 0.19977 \\
\hline 150 & 0.01440 & 0.01240 & 0.05820 & 0.65216 \\
\hline 250 & 0.00850 & 0.00656 & 0.03076 & 1.06932 \\
\hline 300 & 0.00645 & 0.00418 & 0.01963 & 1.16845 \\
\hline \multicolumn{5}{|c|}{ Right Finger } \\
\hline $\operatorname{FREQ}(\mathrm{Hz})$ & $\operatorname{VDT}(\mathrm{mm})$ & S.E. MEAN & S.D. MEAN & Accel(peak) [g] \\
\hline 5 & 0.07800 & 0.02800 & 0.13140 & 0.00393 \\
\hline 10 & 0.11160 & 0.07900 & 0.37070 & 0.02246 \\
\hline 50 & 0.05860 & 0.05130 & 0.24040 & 0.29488 \\
\hline 150 & 0.01680 & 0.01490 & 0.06980 & 0.76085 \\
\hline 250 & 0.00682 & 0.00475 & 0.02228 & 0.85797 \\
\hline 300 & 0.00551 & 0.00326 & 0.01528 & 0.99817 \\
\hline \multicolumn{5}{|c|}{ Left Oral Angle } \\
\hline $\operatorname{FREQ}(\mathrm{Hz})$ & $\operatorname{VDT}(\mathrm{mm})$ & S.E. MEAN & S.D. MEAN & Accel(peak) [g] \\
\hline 5 & 0.47590 & 0.06950 & 0.32580 & 0.02395 \\
\hline 10 & 0.34400 & 0.10100 & 0.47400 & 0.06924 \\
\hline
\end{tabular}




\begin{tabular}{|c|c|c|c|c|}
\hline 50 & 0.19470 & 0.06040 & 0.28320 & 0.97975 \\
\hline 150 & 0.08220 & 0.01470 & 0.06880 & 3.72275 \\
\hline 250 & 0.06072 & 0.00451 & 0.02113 & 7.63872 \\
\hline 300 & 0.04573 & 0.00295 & 0.01385 & 8.28424 \\
\hline \multicolumn{5}{|c|}{ Right Oral Angle } \\
\hline FREQ(Hz) & VDT(mm) & S.E. MEAN & S.D. MEAN & Accel(peak) $[\mathrm{g}]$ \\
\hline 5 & 0.45940 & 0.08210 & 0.38520 & 0.02312 \\
\hline 10 & 0.24890 & 0.06900 & 0.32360 & 0.05010 \\
\hline 50 & 0.17170 & 0.06750 & 0.31640 & 0.86401 \\
\hline 150 & 0.08110 & 0.01430 & 0.06710 & 3.67293 \\
\hline 250 & 0.06269 & 0.00489 & 0.02292 & 7.88656 \\
\hline 300 & 0.04443 & 0.00347 & 0.01628 & 8.04874 \\
\hline
\end{tabular}

Table 4: Adult male $(\mathrm{N}=23)$ VDT descriptive statistics. Acceleration: $a=-2 \Pi 2 \mathrm{f} 2 \mathrm{D} \sin (2 \pi \mathrm{ft})$, where $\mathrm{D}$ is displacement (mm), and $\mathrm{f}$ is frequency $(\mathrm{Hz})$.

\begin{tabular}{|c|c|c|c|c|}
\hline \multicolumn{5}{|c|}{ ADULT MALE } \\
\hline \multicolumn{5}{|c|}{ Left Finger } \\
\hline FREQ(Hz) & $\operatorname{VDT}(\mathrm{mm})$ & S.E. MEAN & S.D. MEAN & Accel(peak) [g] \\
\hline 5 & 0.073000 & 0.015700 & 0.072200 & 0.00367 \\
\hline 10 & 0.030760 & 0.004720 & 0.021620 & 0.00619 \\
\hline 50 & 0.007220 & 0.001090 & 0.005000 & 0.03633 \\
\hline 150 & 0.001954 & 0.000260 & 0.001190 & 0.08849 \\
\hline 250 & 0.001792 & 0.000152 & 0.000696 & 0.22544 \\
\hline 300 & 0.001965 & 0.000204 & 0.000935 & 0.35597 \\
\hline \multicolumn{5}{|c|}{ Right Finger } \\
\hline FREQ(Hz) & $\operatorname{VDT}(\mathrm{mm})$ & S.E. MEAN & S.D. MEAN & Accel(peak) [g] \\
\hline 5 & 0.129300 & 0.044100 & 0.202200 & 0.00651 \\
\hline 10 & 0.063900 & 0.032300 & 0.148200 & 0.01286 \\
\hline 50 & 0.009520 & 0.001200 & 0.005510 & 0.04791 \\
\hline 150 & 0.001937 & 0.000168 & 0.000771 & 0.08772 \\
\hline 250 & 0.004500 & 0.002740 & 0.012540 & 0.56611 \\
\hline 300 & 0.002157 & 0.000326 & 0.001494 & 0.39075 \\
\hline \multicolumn{5}{|c|}{ Left Oral Angle } \\
\hline FREQ(Hz) & $\operatorname{VDT}(\mathrm{mm})$ & S.E. MEAN & S.D. MEAN & Accel(peak) [g] \\
\hline 5 & 0.41600 & 0.06900 & 0.31640 & 0.02093 \\
\hline 10 & 0.26060 & 0.08120 & 0.37210 & 0.05245 \\
\hline 50 & 0.14740 & 0.05730 & 0.26240 & 0.74173 \\
\hline 150 & 0.06552 & 0.00641 & 0.02938 & 2.96733 \\
\hline 250 & 0.06224 & 0.00386 & 0.01767 & 7.82994 \\
\hline 300 & 0.04713 & 0.00237 & 0.01087 & 8.53786 \\
\hline \multicolumn{5}{|c|}{ Right Oral Angle } \\
\hline FREQ(Hz) & $\operatorname{VDT}(\mathrm{mm})$ & S.E. MEAN & S.D. MEAN & Accel(peak) [g] \\
\hline 5 & 0.49400 & 0.07410 & 0.33940 & 0.02486 \\
\hline 10 & 0.20210 & 0.02420 & 0.11090 & 0.04068 \\
\hline 50 & 0.12510 & 0.01560 & 0.07160 & 0.62952 \\
\hline 150 & 0.07974 & 0.00825 & 0.03779 & 3.61134 \\
\hline 250 & 0.05871 & 0.00413 & 0.01894 & 7.38586 \\
\hline 300 & 0.04847 & 0.00274 & 0.01254 & 8.78061 \\
\hline
\end{tabular}


LMM analysis revealed that the effects of sex $(F(1,78)=$ $0.83, \mathrm{p}=0.363)$ and age group $(\mathrm{F}(1,78)=2.11, \mathrm{p}=0.1507)$ on vibrotactile threshold were not significant. However, the effects of skin site $(F(3,1836)=115.74, p<0.0001)$ and stimulus frequency $(F(5,1832)=146.42, p<0.0001)$ were significant. The marginal means estimated for different skin sites and vibrotactile stimulus frequencies are shown in (Figures 6 \& 7), respectively. There was a significant interaction between skin site and stimulus frequency $(F(15,1832)=17.10, p<0.0001)$. Post-hoc pairwise comparisons at a Bonferroni-corrected alpha level further revealed a pattern of differences between VDTs at the lower versus higher stimulus frequencies. For example, in the left glabrous index finger, significant differences were noted for $5 \mathrm{~Hz}$ stimulation versus $150 \mathrm{~Hz}$ (adj. $\mathrm{p}<0.05$ ), $5 \mathrm{~Hz}$ stimulation versus $250 \mathrm{~Hz}$ (adj. $\mathrm{p}<0.05$ ), and $5 \mathrm{~Hz}$ stimulation versus $300 \mathrm{~Hz}$ (adj. $\mathrm{p}<0.05$ ). A similar pattern was found for VDTs in the right glabrous index finger, with highly significant differences between $5 \mathrm{~Hz}$ stimulation and $150 \mathrm{~Hz}$ (adj. $\mathrm{p}$ $<0.01$ ), between $5 \mathrm{~Hz}$ stimulation and $250 \mathrm{~Hz}$ (adj. $\mathrm{p}<0.01$ ), and between $5 \mathrm{~Hz}$ stimulation and $300 \mathrm{~Hz}$ (adj. $\mathrm{p}<0.01$ ).

The profile of post-hoc pairwise comparisons for the face was different from the glabrous index finger as absolute threshold values were higher and the magnitude of VDT differences was greater across stimulation frequencies compared to glabrous finger VDTs. For example, the left oral angle showed significant differences between the $5 \mathrm{~Hz}$ stimulus and all other higher stimulation frequencies $(10,50,150,250$, and $300 \mathrm{~Hz}$; adj. $\mathrm{p}<0.0001)$, and similarly apparent for VDTs at $10 \mathrm{~Hz}$ stimulation versus VDTs obtained at higher stimulation frequencies $(50,150,250$, and 300 Hz; adj. p < 0.0001). The VDT at $50 \mathrm{~Hz}$ stimulation was significantly elevated compared to the VDT at $300 \mathrm{~Hz}$ (adj. p < 0.05). The right oral angle skin site showed significant VDT differences between the $5 \mathrm{~Hz}$ stimulus and all other higher frequency stimulation $(10,50$, 150, 250, and $300 \mathrm{~Hz}$; adj. $\mathrm{p}<0.0001$ ), and significant differences between the $10 \mathrm{~Hz}$ stimulus and higher test frequencies $(150,250$, and $300 \mathrm{~Hz}$; adj. p < 0.0001), and significant differences between the $50 \mathrm{~Hz}$ stimulus and higher test frequencies $(250$, and $300 \mathrm{~Hz}$; adj. $\mathrm{p}<0.01$ ).

Any significant difference in VDTs was found neither between the left and right glabrous finger, nor between left and right oral angle, as a function of stimulus frequency. The pairwise contrasts between hand and face VDTs showed the most significant differences in VDTs at 5, 10, and $50 \mathrm{~Hz}$ (all adj. p < 0.0001).

\section{Discussion}

The efficacy of an automatic SIUD adaptive procedure to estimate VDTs has been shown for the glabrous hand and perioral skin in response to sinusoidal mechanical stimuli presented in randomized blocks at 5, 10, 50,150,250, and 300 Hz for both children and young adults. The resulting VDT function (aka vibrogram) was acquired in approximately 3 minutes 45 seconds for each skin site. An embedded microcontroller provided real-time stimulus generation and randomization of test frequencies, adaptive stimulus amplitude control, threshold response visualization and automated logging of participant threshold measures to an Excel file. VDT's from the present cohort of 45 adults (mean age 23.98 years) are consistent with results from a previous study using the same instrumentation which included a larger cohort of 89 neurotypical adults (mean age 24.33 years) [2].

Collectively, these studies provide strong evidence that VDTs are dependent on stimulus site and frequency of vibrotactile stimulation [17] with no discernable difference between men and women, and no measurable difference in VDTs between left and right skin surfaces. The differences in the VDT's between the hand and face are presumed to reflect the unique typing and distribution of mechanoreceptors and structure of integument in the face and hand. The increased sensitivity (classic U-function) at 250 $\mathrm{Hz}$, attributable to the presence of the rapidly adapting Pacinian corpuscles in the glabrous hand, was not apparent for perioral VDTs sampled from either adults or children. A decrease in VDT at $250 \mathrm{~Hz}$ in the left and right fingers was anticipated due to the presence of Pacinian corpuscles in the hand, but not the lower face $[4,6]$. Across both age groups and sexes, variability in mean VDTs decreased with increases in stimulus frequency.

Additionally, with increasing frequency, the differences in mean VDTs between the hand and lower face decreased. Our 37 neurotypical preadolescent children (age 0 to 13 years), yielded VDT curves which looked very similar to the adult form. This was confirmed by LMM which did not produce a significant main effect for VDT as a function of age group ( $p=0.1507$ ). The lack of a significant main effect on VDTs for age group was not entirely surprising given the precocious development of the human somatosensory system. For example, perioral reflex sensitivity to punctate mechanical stimulation of the lip and lateral face is mediated, in part, by trigeminal primary mechanoreceptive afferents of the maxillary and mandibular branches of $\mathrm{CN}$ V. The mechanically evoked electrophysiological trigeminofacial response shows its most rapid course of development in terms of amplitude and decreased latency during the first year of life to approximate an adult-like response $[18,19]$. This suggests the precocial somatosensory apparatus of the human face (trigeminal sensory branches) is well developed by late infancy. Thus, the child participants in the current study can be expected to have adult-like VDT sensitivity in the hand and face.

The implementation of the SIUD adaptive tracking algorithm [8] for automated vibrotactile threshold estimation worked well with all neurotypical children and adults who participated in this study. The resultant VDT function based on the 6 designated test frequencies was completed in under 4 minutes at each skin site, making it possible to consent participants and bilaterally assess 
their orofacial and glabrous hand tactile sensitivity in less than 30 minutes. The literature provides compelling evidence that VDT assessment is an important and useful tool for investigating early sensory nerve damage as well as central somatosensory perceptual disorders associated with stroke or developmental disorders $[1,2]$. As described in the following sections, numerous clinical populations (industry vibration exposure, drug-induced and diabetic neuropathies, cerebrovascular stroke, neurodevelopmental disorders) with suspect somatosensory function may benefit from VDT studies based on the SIUD adaptive protocol over the extended range of stimulus frequencies ( 5 to $300 \mathrm{~Hz}$ ).

\section{VDTs and Vibration Exposure}

Prolonged exposure of the hand to mechanical vibration at test frequencies ranging from 31.5 to $250 \mathrm{~Hz}$ has been shown to significantly elevate VDTs in the digits of the glabrous hand among young adult males $[20,21]$. Upper limb and whole-body vibration in adults (e.g., 4, 20, 31.5, 100 and $125 \mathrm{~Hz}$ ) also elevated VDTs [22]. Elevated VDTs were greatest after simultaneous exposure to wholebody and hand-arm vibration. The increase in VDTs following exposure to both types of vibration was greater than that following either type of vibration separately for all frequencies [22]. Elevated VDTs resulting from prolonged exposure to vibration may reflect changes in the dynamics of blood flow to the cutaneous sensory surface. For example, [23]. examined the potential association between VDTs in the Pacinian channel and decreases in finger blood flow caused by vibration in 40 healthy adult participants $(20 \mathrm{~F} / 20 \mathrm{M})$. Blood flow in the fingers was evaluated using a straingage plethysmographic method (measured in D3 in both hands).

An HVLab Vibrotactile Perception Meter was used to determine VDTs of the hand at $125 \mathrm{~Hz}$ using a von Békésy threshold tracking algorithm. This study found that blood flow was reduced in the fingers of the hand that received $125 \mathrm{~Hz}$ vibration and the contralateral hand which was not exposed to this vibration. Thus, the Pacinian corpuscles might contribute to vibration-induced vasoconstriction. Sex was a significant factor, with women manifesting greater reductions in blood flow in the fingers attributed to vibration exposure [23]. The vibratory contactor size (3 vs $6 \mathrm{~mm}$ diameter) was also positively related to the magnitude of VDT elevation following vibration exposure to the hand [24].

\section{VDTs and Cerebrovascular Stroke}

Clinical application of automated VDT measurement has found application in survivors of cerebrovascular stroke who present movement disorders and changes in somatosensory function that may be inconspicuous and difficult to measure. In a recent report, 7 adults who sustained unilateral middle cerebral artery ischemic strokes were assessed using the SIUD adaptive threshold tracking procedure to determine VDTs for the glabrous index finger and perioral hairy skin in response to vibrotactile stimuli presented at $5,10,50,150,250$, and $300 \mathrm{~Hz}$ [9]. Linear mixed modeling revealed significant main effects for stroke status, structure and vibrotactile frequency. As expected, VDTs were dependent on test frequency, and skin site, with VDTs significantly higher for the oral angle compared to the glabrous index finger. Stroke patients had significantly higher VDTs than normal adults. The secondary analyses revealed significant differences between the neurotypical oral angle VDTs and both the contralesional and ipsilesional oral angle VDTs. Finally, the contralesional index finger manifest significantly higher VDTs than the ipsilesional index finger. This automated adaptive VDT tracking algorithm shows great promise in providing clinicians with a reliable tool for rapid assessment of the cutaneous somatosensory system on both glabrous and hairy skin in stroke survivors to objectively assess neurosensory status.

\section{VDTs and Metabolic Disorders}

Select metabolic disorders, including diabetes, may lead to progressive peripheral neuropathy and impaired vibrotactile sensitivity. In adults, digit VDTs are notably elevated, especially at higher test frequencies (e.g., $250 \& 500 \mathrm{~Hz}$ ) with Type I and Type II diabetes $[10,11]$. In children with Type I diabetes, (Isling, Dahlin, and Larsson, 2018) 13 of 72 participants showed diminished vibrotactile sensation on the sole of the foot, with 3 of the 13 subjects had decreased vibrotactile sensation in both the foot and the hand. One participant had decreased sensation of the hand only. VDT assessment shows promise as a tool to evaluate the onset and progression of diabetic neuropathy in adults and children [15].

\section{VDTs in Normal and Atypical Development}

There is growing interest in understanding somatosensory function in children. For example, tactilometry based on the method of limits was used to assess VDTs for two digits of the hand (glabrous D5, D2) at 8, 16, 64, 125, 250, and $500 \mathrm{~Hz}$ in 269 neurotypical children (age 8 to 20 years) using a VibroSense Meter ${ }^{\circledR}$ device in which the sinusoidal threshold stimulus amplitude is expressed as acceleration in $\mathrm{dB}$ relative to $0.00001 \mathrm{~m} / \mathrm{s}^{2}$ [15]. Digit VDTs referenced to acceleration increased as frequency increased (with the exception of 125 and $64 \mathrm{~Hz}$ ). Older participants manifest lower thresholds than younger participants. Elevated VDTs and changes in tactile adaptation have been reported in schoolage children (age 8-12 years) with autism spectrum disorder (ASD) $[14,25]$. Psychophysical detection of the rate of amplitude modulation of tactile stimuli discriminates ASD from neurotypical control children [26]. Children (ages 7-18 years) with obsessive compulsive disorder showed impaired amplitude discrimination adaptation and elevated just noticeable differences (JNDs) to tactile stimuli (25Hz vibration) delivered to the fingertips on the nondominant hand [13].

Males showed a disproportionately greater impairment in these tactile processing measures. One limitation of the present 
study is the relatively small number of participants in the child cohorts, with substantially fewer female children compared to their male counterparts. We plan to extend VDT studies to include clinical populations at risk for peripheral neuropathy and central somatosensory dysfunction, and younger children, perhaps as young as 6 years of age. We anticipate that younger children will be able to complete the SIUD psychophysical sensory tactile tracking paradigm which is similar in many respects to pure tone audiometry which children can complete beginning around the age of 6 years.

\section{Conclusion}

The present study examined vibrotactile detection thresholds in cohorts of neurotypical children and adults. LMM revealed significant main effects for stimulus frequency, skin site, but not for age group, and sex. Overall, VDTs were higher at the oral angle compared to the glabrous hand, and the classic $250 \mathrm{~Hz}$ PC dip was absent at the oral angle in children and adults. As frequency increased, the differences in mean VDT between the oral angles and index fingers decreased. The automated SIUD VDT tracking protocol and mechanical stimulus control system provides clinical investigators with a reliable tool for rapid assessment of somatosensory system on both glabrous and hairy skin in humans across the lifespan.

\section{Author Contribution}

E.S. (conceptualization, data collection, data analysis, manuscript preparation), J.L. (data analysis, statistical modeling, edit manuscript draft), J.G. (engineering and instrument design, data acquisition, edit draft). S.B. (conceptualization, study design, instrument design, data analysis, manuscript preparation and editing, secure internal funding), All authors have read and agreed to the published version of the manuscript.

\section{Funding}

This research received no external funding.

\section{Acknowledgment}

This study was supported in part by the Barkley Trust Foundation (Barlow). Gratitude is expressed to Emma Loberg and Alyssa Molina for technical assistance.

\section{Conflicts of Interest}

The authors confirm that there are no conflicts of interest associated with this publication and there has been no financial support for this work that could have influenced its outcome.

\section{References}

1. Gandhi MS, Sesek R, Tuckett R, Morris Bamberg (2011) Progress in vibrotactile threshold evaluation techniques: a review. Journal Hand Therapy 24: 240-256.
2. Barlow SM, Custead R (2019) Vibrography: Single-Interval Up/Down (SIUD) Adaptive Vibrotactile Threshold Estimation of the Glabrous Hand and Perioral Face in Neurotypical Adults. Biomedical J Sci \& Tech Research 22(4): 16837-16847.

3. Venkatesan L, Barlow SM, Kieweg D (2015) Age-and sex-related changes in vibrotactile sensitivity of hand and face in neurotypical adults. Somatosensory \& Motor Research 32(1): 44-50.

4. Barlow SM (1987) Mechanical frequency detection thresholds in the human face. Experimental Neurology 96(2): 253-261.

5. Verrillo RT (1966) Vibrotactile sensitivity and the frequency response of the Pacinian corpuscle. Psychonomic Science 4(1): 135-136.

6. Munger BL, Halata Z (1983) The sensory innervation of primate facial skin. I. Hairy skin. Brain Res Rev 5(1): 45-80.

7. Andreatta RD, Davidow JH (2006) Mechanical frequency and stimulationsite-related differences in vibrotactile detection capacity along the lip vermilion in young adults. Clinical Oral Investigations 10(1): 17-22.

8. Lecluyse W, Meddis R (2009) A simple single-interval adaptive procedure for estimating thresholds in normal and impaired listeners. J Acoustical Society America 126(5): 2570-2579.

9. Barlow SM, Custead R, Lee J (2019) Adaptive vibrotactile threshold estimation of the glabrous hand and perioral face following MCA stroke. Biomedical J Sci \& Technical Research 23(3): 2019.

10. Dahlin LB, Granberg V, Rolandsson O, Rosén I, Dahlin E, et al. (2011) Disturbed vibrotactile sense in finger pulps in patients with Type 1 diabetes-correlations with glycaemic level, clinical examination and electrophysiology. Diabetic Medicine 28(9): 1045-1052.

11. Dahlin LB, Thrainsdottir S, Cederlund R, Thomsen NOB, Eriksson KF, et al. (2008) Vibrotactile sense in median and ulnar nerve innervated fingers of men with Type 2 diabetes, normal or impaired glucose tolerance. Diabetic Medicine 25(5): 543-549.

12. Ising E, Dahlin LB, Larsson HE (2018) Impaired vibrotactile sense in children and adolescents with type 1 diabetes-Signs of peripheral neuropathy. PloS one 13(4): e0196243.

13. Güçlü B, Tanıdır C, Canayaz E, Güner B, İpek Toz H, et al. (2015) Tactile processing in children and adolescents with obsessive-compulsive disorder. Somatosensory \& Motor Research 32(3): 163-171.

14. Tavassoli T, Bellesheim K, Tommerdahl M, Holden JM, Kolevzon A, et al. (2016) Altered tactile processing in children with autism spectrum disorder. Autism Research 9(6): 616-620.

15. Dahlin LB, Güner N, Larsson HE, Speidel T (2015) Vibrotactile perception in finger pulps and in the sole of the foot in healthy subjects among children or adolescents. PloS one 10(4): e0119753.

16. SAS Institute. SAS/STAT 9.4 user's guide. Cary, NC: SAS Institute Inc 2002-2012.

17. Forta NG, Griffin MJ, Morioka M (2012) Vibrotactile difference thresholds: Effects of vibration frequency, vibration magnitude, contact area, and body location. Somatosensory \& Motor Research 29(1): 28-37.

18. Barlow SM, Finan S, Rowland SG (1992) Mechanically evoked perioral reflexes in infants. Brain Research 599: 158-160.

19. Barlow SM, Finan DS, Bradford PT, Andreatta R (1993) Transitional properties of the mechanically evoked perioral reflex from infancy through adulthood. Brain Research 623(2): 181-188.

20. Burström L, Lundström R, Hagberg M, Nilsson T (2009) Vibrotactile perception and effects of short-term exposure to hand-arm vibration. Annals Occupational Hygiene 53(5): 539-547.

21. Mahbub MH, Ohnari H, Tanigawa K, Hiroshige $\mathrm{K}$, Takahashi $\mathrm{Y}$, et al (2011) Vibrotactile perception at glabrous and nonglabrous skin of fingers: repeatability of measurements and changes induced by acute vibration exposure. J Occupational Health 53(1): 10-15. 
22. Kowalski P, Zając J (2012) Research on Simultaneous Impact of HandArm and Whole-Body Vibration. Int J Occupational Safety Ergonomics 18(1): 59-66

23. Ye Y, Griffin MJ (2011) Reductions in finger blood flow in men and women induced by $125-\mathrm{Hz}$ vibration: association with vibration perception thresholds. J Applied Physiology 111(6): 1606-1613.

24. Ye Y, Griffin MJ (2013) Reductions in finger blood flow induced by 125 $\mathrm{Hz}$ vibration: effect of area of contact with vibration. European J Applied Physiology 113(4): 1017-1026.

ISSN: 2574-1241

DOI: 10.26717/BJSTR.2021.35.005768

Steven M Barlow. Biomed J Sci \& Tech Res

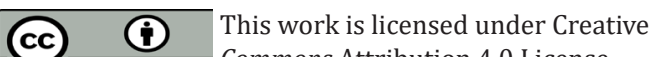
BY Commons Attribution 4.0 License

Submission Link: https://biomedres.us/submit-manuscript.php
25. Puts NA, Wodka EL, Tommerdahl M, Mostofsky SH, Edden RA (2014) Impaired tactile processing in children with autism spectrum disorder. J Neurophysiology 111(9): 1803-1811.

26. Francisco E, Holden J, Zhang Z, Favorov O, Tommerdahl M (2011) Rate dependency of vibrotactile stimulus modulation. Brain Research 415: 76-83.

$\begin{array}{ll}\text { BIOMEDICAL } & \text { Assets of Publishing with us } \\ \text { RESEARCHES } & \text { - Global archiving of articles } \\ \text { - Immediate, unrestricted online access }\end{array}$

